\title{
Reacciones adversas psiquiátricas asociadas a nuevos macrólidos. A propósito de tres casos
}

\author{
MP. Sanz de Miguela , E. Sancho Gracia a , B. Chapi Peña ${ }^{a}$, A. Campos Bernal, \\ R. Romero Gil ${ }^{\mathrm{a}}$, C. García Vera ${ }^{\mathrm{b}}$ \\ aMIR-Pediatría. Hospital Infantil Universitario Miguel Servet. Zaragoza. España. \\ bPediatra. CS Sagasta-Ruiseñores. Instituto Aragonés de Ciencias de la Salud. Zaragoza. España.
}

\section{Resumen}

Los macrólidos representan el 10-15\% del mercado mundial de antibióticos orales. Son uno de los grupos de antibióticos más seguros; las reacciones adversas graves son muy raras. Pueden producir reacciones gastrointestinales, hepatotoxicidad y ototoxicidad. Además, las reacciones psiquiátricas se encuentran entre los efectos adversos esporádicamente asociados con su administración, aunque su mecanismo no está bien establecido.

Casos descritos en la Food and Drug Administration (FDA) de EE.UU. muestran que la claritromicina y el ciprofloxacino son los antibióticos más frecuentemente asociados con el desarrollo de manía. Este término ha sido denominado antibiomanía.

Presentamos tres casos clínicos vistos en una consulta de Atención Primaria en el período de un año con cuadros similares de hiperactividad y agresividad, coincidiendo con la administración de antibióticos de la familia de los macrólidos.

Palabras clave: Macrólidos. Reacción adversa. Manía.

Psychiatric adverse reactions associated to new macrolides. Three case reports

\section{Abstract}

Macrolides represent the $10-15 \%$ of the world-wide market of oral antibiotics. They are one of the safer groups of antibiotics, being the severe adverse reactions very rare. They can produce gastrointestinal reactions, hepatotoxicity and ototoxicity. The psychiatric reactions are found sporadically among the adverse effects.

Cases reported to the FDA showed that clarithromycin and ciprofloxacin are the most frequent antibiotics associated with the development of mania. The syndrome has been termed antibiomania.

We present three clinical cases seen in a Primary Care office in the last year with similar pictures of hyperactivity and aggressiveness coinciding with the administration of antibiotics of the family of the macrolides.

Key words: Macrolides. Adverse reaction. Mania.

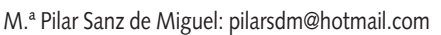

Los autores declaran no presentar conflictos de intereses en relación con la preparación y publicación de este artículo.

Trabajo presentado en la modalidad de comunicación/póster en la 5. ${ }^{a}$ Reunión Anual de la AEPap y 5. ${ }^{a}$ Reunión de la ArAPAP en Zaragoza, el 13 y 14 de noviembre de 2009. 


\section{Introducción}

Los macrólidos representan el 10 $15 \%$ del mercado mundial de antibióticos orales. El primer antibiótico de la familia de los macrólidos fue la eritromicina, que se introdujo a principios de los años cincuenta. La troleandomicina, en desuso actualmente, y la espiramicina son otros fármacos de este grupo con largos años de comercialización. Posteriormente se introdujeron nuevos fármacos: diritromicina, midecamicina, miocamicina, josamicina y roxitromicina, con un espectro de actividad antibacteriana muy similar al de la eritromicina, pero con variaciones en la farmacocinética, en el potencial de interacciones y en la tolerancia gástrica. Más recientemente se han desarrollado nuevos macrólidos, como la azitromicina y la claritromicina, que añaden además diferencias en el espectro de acción antibacteriana, lo que ha promovido su utilización en otras indicaciones, como en infecciones por micobacterias atípicas en pacientes con infección por VIH y en la erradicación de H. pylori'.

Son uno de los grupos de antibióticos más seguros y las reacciones adversas graves son muy raras. Las reacciones adversas más frecuentes son las gastrointestinales, que suponen el $15-20 \%$ en el caso de la eritromicina y el $5 \%$ en el de los nuevos macrólidos. La intolerancia gástrica puede ser un reflejo de su efecto estimulante sobre la motilidad intestinal, debido a la liberación endógena de motilina. La hepatotoxicidad está relacionada con la capacidad de algunos macrólidos de inducir nitrosoalcanos. La frecuencia de aparición de hepatitis colestática con eritromicina se ha estimado en un 2-4\%; el potencial hepatotóxico de otros macrólidos es menor ${ }^{2}$.

La aparición de ototoxicidad está bien establecida aunque es muy rara; la función auditiva es la más afectada en relación únicamente con dosis elevadas y es generalmente reversible.

Las reacciones psiquiátricas se encuentran entre los efectos adversos esporádicamente asociados con su administración, aunque su relación causal y su mecanismo no están bien establecidos. Los primeros casos no se publicaron hasta 30 años después de su comercialización. Los síntomas descritos en los casos asociados a eritromicina son confusión, paranoia, alucinaciones visuales, temor, falta de control y pesadillas. Dichos efectos se han visto incluso con dosis convencionales en las 12-24 horas desde el inicio del tratamiento con resolución en los días siguientes de la retirada o de la reducción de la dosis al $50 \%$. 
En concreto, en la ficha técnica de la claritromicina figuran los siguientes efectos adversos psiquiátricos: ansiedad, insomnio, pesadillas, confusión y alucinaciones (con una frecuencia de entre $1 / 100$ y $1 / 1.000$ pacientes) y desorientación, psicosis y despersonalización (entre $1 / 1.000$ y $1 / 10.000$ pacientes). No consta el tipo de psicosis que se ha descrito con el fármaco y no figuran específicamente los episodios de manía ${ }^{3}$.

En la base de datos nacional de reacciones adversas a medicamentos (FEDRA) hasta junio de 2008 se habían recogido 9 casos, notificados espontáneamente, de episodios maníacos tras la toma de claritromicina. La edad de los pacientes variaba entre 45 y 73 años, con una mediana de 62 años; 7 eran mujeres y 2 hombres, y no existía, por tanto, ningún caso en la infancia ${ }^{4}$.

Revisada la bibliografía, Fidan ${ }^{5}$ documenta el caso de un niño de 12 años que presenta un episodio de insomnio, verborrea, alucinaciones y delirios de grandeza, coincidiendo con que el paciente estaba en tratamiento con claritromicina por una neumonía, por lo que cumplía todos los criterios del Manual Diagnóstico y Estadístico de los Trastornos Mentales (DSM-IV) para el diagnóstico de manía. Dicho paciente no presentaba una historia familiar de antecedentes psiquiátricos, y los datos de laboratorio y las pruebas de neuroimagen fueron normales. Comenzó con los síntomas tras la primera dosis.

Casos descritos por la Food and Drug Administration estadounidense muestran que la claritromicina y el ciprofloxacino son los antibióticos más frecuentemente asociados con el desarrollo de manía. A esta manifestación clínica incluso se le ha dado la denominación de antibiomanía ${ }^{6}$.

\section{Casos clínicos}

Con respecto al primer caso clínico, se trata de un varón de 4 años en tratamiento con claritromicina solución en dosis recomendada, por un cuadro de faringoamigdalitis. Acude a nuestra consulta porque sus padres le notan un comportamiento irritable y violento 1-2 horas después de cada dosis administrada de dicho antibiótico, si bien con las sucesivas dosis la clínica ha ido disminuyendo. Se le suspende el antibiótico y no vuelve a presentar dicha sintomatología.

El segundo paciente es un varón de 3 años que acude por un cuadro de ronquera sin fiebre habiéndosele prescrito acitromicina en urgencias en dosis habituales. A lo largo de la consulta, los padres nos comentan que estuvo particularmente agresivo durante ese período, gritando y pegando a su padre, clínica 
que fue mejorando paulatinamente. No estaba tomando ninguna medicación concomitante.

El tercer caso es también un varón de 2 años con un cuadro catarral acompañado de fiebre al que en urgencias se le prescribe azitromicina jarabe en dosis habituales, con el diagnóstico de otitis media y traqueobronquitis. Nos consultan tres días después por agresividad (pega a su madre y a su hermana), acompañada de despertares nocturnos, con irritabilidad y pesadillas. El paciente estaba tomando simultáneamente terbutalina, si bien dicha medicación había sido tomada con anterioridad sin referir dicha sintomatología. Paulatinamente cede la agresividad y desaparece al $7 .^{\circ}-8 .^{\circ}$ día.

Todos los casos fueron declarados al servicio de farmacovigilancia y aceptados como reacción adversa a medicamentos.

\section{Discusión}

Nos hallamos ante tres casos clínicos de reacciones adversas psiquiátricas producidas por fármacos de la familia de los macrólidos. Cuando en la consulta nos encontremos ante el caso más florido que nos pueden dar estos fármacos, la manía, extremadamente rara en pacientes prepúberes, deberemos diferenciarla de causas orgánicas como epilepsias de lóbulo temporal, hipertiroidismo, esclerosis múltiple, lupus eritematoso o enfermedad de Wilson, u otras causas farmacológicas como la ingesta de antidepresivos tricíclicos, inhibidores de la recaptación de serotonina, simpaticomiméticos y corticoides, que, como se sabe, pueden exacerbar los síntomas maníacos.

No obstante, la mayoría de casos producidos en la infancia por macrólidos presentan una clínica más larvada de hiperactividad y agresividad sin llegar a cumplir los criterios del DMS-IV para el diagnóstico de manía, motivo por el cual probablemente se trate de un problema infradiagnosticado.

El mecanismo por el que estos fármacos inducen cuadros de este tipo es desconocido. Existen varias hipótesis. Manev postula que los antibióticos de la familia de los macrólidos pueden producir síntomas maníacos a través de su acción sobre la vía inhibitoria de la neurotransmisión glutaminérgica en el cerebro ${ }^{7}$. Se sabe que la difusión de los macrólidos en el sistema cerebroespinal es pobre -por ejemplo, el pico de concentración de claritromicina en el líquido cefalorraquídeo (LCR) es menos del $1 \%$ de la concentración sérica-, aunque en conejos a los que experimentalmente se les induce meningitis, las cifras de 
concentración LCR/concentración sérica son del $25-45 \%$, dependiendo de las dosis administradas. No obstante, la claritromicina experimentalmente es eficaz para el tratamiento de la encefalitis por toxoplasma, y algunos macrólidos antihelmínticos como la ivermectina se unen en el córtex cerebral y cerebelo de los mamíferos a canales asociados al complejo del canal de cloro GABA-benzodiazepina mejorando la actividad del diazepam in vivo. Sin embargo, cualquier sugerencia del mecanismo involucrado en la inducción de anomalías del comportamiento es especulativa1.

Aunque las reacciones psiquiátricas por macrólidos son poco frecuentes, re- sulta importante conocer su existencia a la hora de su prescripción y presentar un alto umbral de sospecha, ya que están infradiagnosticadas. Nuestros tres casos sugerían a los macrólidos como causa potencial de cuadros psicóticos, como efectivamente fue confirmado por el servicio de farmacovigilancia.

Es de destacar, en este sentido, la importancia de poner dichas reacciones adversas en conocimiento del sistema de farmacovigilancia, encargado de identificar los riesgos de los fármacos comercializados, para lograr un conocimiento exhaustivo de la incidencia real de los efectos adversos de los fármacos tras la comercialización.

\section{Bibliografía}

1. Gómez-Gil E, García F, Pintor L, Martínez JA, Mensa J, De Pablo J. Clarithromycin-induced acute psychoses in peptic ulcer disease. Eur J Clin Microbiol Infect Dis. 1999;18:70-1.

2. Hassan-Alin $M$, Andersson $T$, Niazi $M$, Röhss K. Studies on drug interaction between esomeprazole, amoxicilin and clarithromycin in healthy subjets. Int I Clin Pharmacol Ther. 2006;44:119-27.

3. Reacciones adversas psiquiátricas asociadas a macrólidos. Boletín RAM. 1997;5(2):5-8 [consultado el 13/02/2010]. Disponible en www.seguridad medicamento.sanidadmadrid.org/RAM/vol-5/Vo lumen $\% 205 \% 20 \mathrm{~N}-2 \% 20 . p d f$
4. Ortiz Domínguez A, Berlanga $C$, Gutiérrez Mora D. A case of clarithromycin-induced manic episode (antibiomania). Int J Neuropsycopharmacol. 2004;7:99-100.

5. Fidan T, Fidan V. Clarithromycin-induced mania in a child. Int J Clin Pharmacol Ther. 2009;47(6):402-4.

6. Abogues A, Stone C, Hobbs WR. Antimicrobial-induced mania (antibiomania) a review of spontaneous report. J Clin Psychopharmacol. 2002;22:71-81.

7. Manev H, Favaron M. Macrolide antibiotics protect neurons in culture against the $\mathrm{N}$-methylD- aspartate receptor-mediated toxicity of glutamate. Brain Res. 1993;624:331-5. 